\title{
Rare Metabolic Syndromes, a Vascular Hypotheses of Alzheimer's Disease, and Drug Developments for Attention and Trauma
}

By Eric Hollander, MD

This month's CNS Spectrums examines the presentation of developmental disorders in adulthood; the role of vascular pathology in "non-vascular" forms of dementia; and the use of prodrug stimulants in childhood attentiondeficit/hyperactivity disorder and glutamate approaches in trauma.

Glutamatergic agents such as lamotrigine have been used successfully for the treatment of posttraumatic stress disorder (PTSD). They could be potentially acting through the stabilization of the corticotropin-releasing factor (CRF) systems. Jyotsna Nair, MD, and Sarbjot Singh Ajit, $M D$ review how glutamate mediates $C R F$ release in various brain regions involved in the pathophysiology of PTSD, and how glutamate modulating agents could stabilize the CRF system and, thereby, improve the symptom complex of PTSD (reexperiencing, hyperarousal, and avoidance). The role of glutamate and CRF in PTSD and other anxiety disorders are still being elucidated; however, it is clear that the glutamatergic systems play a role in the pathophysiology of PTSD. The role of glutamate in anxiety and depression, refractory states, and especially in trauma, is particularly timely, and may form the basis for new drug development in these areas.

Succinic semialdehyde dehydrogenase (SSADH) deficiency is a rare neurometabolic disorder of $\gamma$-aminobutyric acid degradation. While neurologic manifestations such as developmental delay are typical during infancy, limited data are available on adolescent and adult symptomatology. Ina Knerr, MD, and colleagues studied 33 adolescents and adults with SSADH deficiency. Mean age at first symptoms was 11 months, but mean age at diagnosis was 6.6 years. Presenting symptoms included motor delay, hypotonia, speech delay, autistic features, seizures, and ataxia. Eighty-two percent demonstrated behavioral problems, such as attention-deficit, hyperactivity, anxiety, or aggression, $33 \%$ had $\geq 3$ behavior problems. Electroencephalograms showed background slowing or epileptiform discharges in $40 \%$ of patients. The variable phenotype in SSADH deficiency suggests the likelihood that this disease may be under-diagnosed. Families of patients with SSADH deficiency should be counseled and supported regarding the anticipated persistence of various neuropsychiatric symptoms into adulthood. Rare metabolic syndromes are of particular interest as causes of developmental disorders, and while well known in neurology, they are rarely diagnosed in psychiatric practice. Also, the presentation of most developmental disorders in adulthood are not well understood, and certainly understudied. This may be because pediatric psychiatrists and neurologists frequently do not treat adults. Adult psychiatrists and neurologists have not received special training and do not treat patients with developmental disabilities. Thus, the gap in knowledge and availability of treatment for adults with developmental disabilities is especially acute.

Current evidence from epidemiological, neuroimaging, pathological, pharmacotherapeutic, and clinical studies indicate an association of

Dr. Hollander is the editor of this journal, Esther and Joseph Klingenstein Professor and Chairman of Psychiatry at the Mount Sinai School of Medicine, and director of the Seaver and New York Autism Center of Excellence in New York City. 
Alzheimer's disease with risk factors of vascular atherosclerotic disease either in isolation or in aggregate. "The metabolic syndrome" is the name for a clustering of risk factors for cardiovascular disease and type 2 diabetes that are of metabolic origin. These include central obesity, elevated plasma glucose, high blood pressure, atherogenic dyslipidemia, a prothrombotic state, and a proinflammatory state. Sotirios Giannopoulos, MD, DSc, and colleagues review the literature with regard to the relationship of Alzheimer's disease with metabolic syndrome. Accumulating evidence suggests a "vascular hypothesis" to be related to the pathology of Alzheimer's disease. In light of this evidence, clinicians may consider lifestyle interventions toward early and effective cardiovascular risk-factor management to reduce the "cardiometabolic" and the "cognitive decline" risk, while further research of other preventive strategies may be warranted. Clearly, both vascular and Alzheimer's type dementias are well known. However, if this hypothesis is borne out through further prospective study, then it could provide an additional potential therapeutic point of intervention and drug discovery for the growing public health problem of Alzheimer's disease.

Lisdexamfetamine dimesylate (LDX), a prodrug stimulant, is indicated for ADHD in children. In short-term studies, once-daily LDX provided efficacy throughout the day. Robert $\mathrm{L}$. Findling, MD, and colleagues assessed the long-term safety, tolerability, and effectiveness of LDX in 6- to 12-year-olds with ADHD via an open-label, multicenter, single-arm study. Of 272 subjects receiving LDX, 147 completed the study. Most adverse events were mild to moderate and occurred during the first 4 weeks. From baseline to endpoint, mean ADHD Rating Scale scores significantly improved. On the Clinical Global Impression-Improvement scale, $>80 \%$ of subjects at endpoint and $>95 \%$ of completers at 12 months were rated "improved." Long-term LDX was generally well tolerated and effective in children with ADHD. Limitations of open-label studies are known, but long-term follow-up data of prodrug stimulants are useful. Evaluating the long-term benefits and risks of prodrug stimulants versus stimulants also require such longterm data.

Thus, the presentation of developmental disorders in adulthood is especially misunderstood, and the role of vascular pathology in "non-vascular" forms of dementia is not fully appreciated. Also, the risks versus benefits of prodrug stimulants in childhood ADHD, and role of glutamate in trauma, is not fully known. This month's CNS Spectrums addresses some of these gaps in our knowledge. May this issue be a welcome companion to your summer reading. CNS 